\title{
Hippocampal Volume in Healthy Controls Given 3-Day Stress Doses of Hydrocortisone
}

\author{
E Sherwood Brown*,', Haekyung Jeon-Slaughter', Hanzhang Lu'², Rhoda Jamadar', Sruthy Issac', \\ Mujeeb Shad', Daren Denniston', Carol Tamminga', Alyson Nakamura' and Binu P Thomas ${ }^{2}$ \\ 'Department of Psychiatry, The University of Texas Southwestern Medical Center, Dallas, TX, USA; ${ }^{2}$ The Advanced Imaging Research Center, \\ The University of Texas Southwestern Medical Center, Dallas, TX, USA
}

\begin{abstract}
In animal models, corticosterone elevations are associated with hippocampal changes that can be prevented with phenytoin. In humans, Cushing's syndrome and long-term prescription corticosteroid use are associated with a reduction in the hippocampal volume. However, little is known about the effects of short-term corticosteroid administration on the hippocampus. The current report examines changes in the hippocampal volume during a brief hydrocortisone exposure and whether volumetric changes can be blocked by phenytoin. A randomized, double-blind, placebo-controlled, within-subject crossover study was conducted in healthy adults $(n=17)$. Participants received hydrocortisone $(160 \mathrm{mg} /$ day)/placebo, phenytoin/placebo, both medications together, or placebo/placebo, with 21 -day washouts between the conditions. Structural MRI scans and cortisol levels were obtained following each medication condition. No significant difference in the total brain volume was observed with hydrocortisone. However, hydrocortisone was associated with a significant $1.69 \%$ reduction in the total hippocampal volume compared with placebo. Phenytoin blocked the volume reduction associated with hydrocortisone. Reduction in hippocampal volume correlated with the change in cortisol levels $(r=-0.58, P=0.03)$. To our knowledge, this is the first report of structural hippocampal changes with brief corticosteroid exposure. The correlation between the change in hippocampal volume and cortisol level suggests that the volume changes are related to cortisol elevation. Although the findings from this pilot study need replication, they suggest that the reductions in hippocampal volume occur even during brief exposure to corticosteroids, and that hippocampal changes can, as in animal models, be blocked by phenytoin. The results may have implications both for understanding the response of the hippocampus to stress as well as for patients receiving prescription corticosteroids. Neuropsychopharmacology (2015) 40, I216-1221; doi:10.1038/npp.2014.307; published online 17 December 20I4
\end{abstract}

\section{INTRODUCTION}

The hippocampus has a high concentration of glucocorticoid (GR) receptors and appears to be a primary target for corticosteroids in the brain (Brown, 2009). Exposure to high levels of corticosteroids in stress paradigms or with corticosterone administration is associated with changes in the hippocampus, including dendritic remodeling, in animal models (Magarinos et al, 1997; Vyas et al, 2002). In non-human primates, most (Sapolsky et al, 1990; Uno et al, 1994; Uno et al, 1989), but not all (Leverenz et al, 1999), studies suggest that exogenous corticosteroid administration is associated with changes in the hippocampus.

In humans, Cushing's disease with elevated cortisol levels due to ACTH-secreting tumors is associated with hippocampal volume reduction and memory impairment (Starkman et al, 1992). Chronic exogenous corticosteroid use is also

*Correspondence: Dr ES Brown, Department of Psychiatry, Psychoneuroendocrine Research Program, The University of Texas Southwestern Medical Center at Dallas, 5323 Harry Hines Boulevard, MC 8849, Dallas, TX75390-8849, USA, Tel: + I 214645 6950, Fax: + | 214 645695 I, E-mail: Sherwood.Brown@UTSouthwestern.edu Received 26 September 2014; revised 31 October 2014; accepted 4 November 20।4; accepted article preview online 20 November 2014 associated with structural and functional hippocampal changes. We reported that patients receiving prescription corticosteroid therapy for a mean of $\sim 8$ years had poorer declarative memory, decreased hippocampal volume, and decreased temporal lobe levels of $N$-acetyl aspartate compared with the controls with similar medical histories not receiving corticosteroids (Brown et al, 2004).

Minimal data are available on the effects of brief exposure to corticosteroids on the human hippocampus. Tessner et al (2007) found no change in hippocampal volume in 14 healthy male controls given a single dose of hydrocortisone $(100 \mathrm{mg})$ or placebo in a crossover fashion. Scheel et al (2010) also reported no difference in hippocampal volume in 21 healthy men receiving 4 days of hydrocortisone (160 mg/day).

We previously reported a trend toward a decline in the declarative memory and a decrease in the hippocampal activation during a task with 3 days of hydrocortisone exposure in a sample of healthy men and women (Brown et al, 2013). The deleterious memory effect of the hydrocortisone $(160 \mathrm{mg} /$ day) was blocked by phenytoin pretreatment. In the current report, the primary aim was to examine the impact of 3 days of hydrocortisone on hippocampal volume and to determine whether phenytoin pretreatment blocks 
any observed effects on the volume. The secondary aim was to explore the relationship between the changes in cortisol levels and changes in brain volume.

\section{METHODS}

The University of Texas Southwestern institutional review board (IRB) approved this study. All participants completed an IRB-approved written informed consent process at the Psychoneuroendocrine Research Program offices on the UT Southwestern campus. The study was registered at http://clinicaltrials.gov (NCT00591006).

Healthy volunteers $(n=17)$ were recruited through flyers and other forms of advertising. Included were men and women aged 18-50, vision corrected to at least $20-40$, education of $\geqslant 12$ years, and normal baseline memory. Excluded were those with a history of brain diseases, taking CNS-acting medications, having contraindications to phenytoin, hydrocortisone or MRI, significant medical conditions, vulnerable populations, or cognitive disorders, baseline 17item HSRD (Hamilton Rating Scale for Depression; Hamilton, 1960) score $>7$ (to exclude those with clinically significant depressive symptoms at baseline), current suicidal ideation or history of a suicide attempt, and history of systemic or past 14-day inhaled corticosteroid use.

Participants and all persons (eg, raters) with participant contact were blinded to the treatment order. The trial ended when the enrollment goal was achieved. The Structured Clinical Interview for DSM-IV (SCID; First et al, 1995) was used to rule out exclusionary psychiatric illnesses.

Participants received each of the four medication conditions (hydrocortisone/placebo, phenytoin/placebo, hydrocortisone/phenytoin, placebo/placebo) and four fMRI scans using a randomized, using a within-subject, crossover design with a 21-day washout between the medication conditions. Medications and placebo identical in appearance were purchased from Abrams Royal Pharmacy, Dallas, Texas. Three days before MRI scans, participants took four capsules containing phenytoin (100 mg) or identical placebo by mouth at $2100 \mathrm{~h}$ ( $400 \mathrm{mg} /$ day). Beginning 2 days before the MRI scans (day after initiating phenytoin or placebo), participants began taking four tablets containing hydrocortisone $(20 \mathrm{mg})$ or placebo at $0900 \mathrm{~h}$ and $2100 \mathrm{~h}(160 \mathrm{mg} /$ day $)$ with the final dose at $0900 \mathrm{~h}$ on the day of imaging. Thus, hydrocortisone was administered for a total of 2.5 days. The dose of hydrocortisone was the same as that used in a memory study (Newcomer et al, 1999) and designed to achieve a stress level of cortisol. Neuroimaging was performed at $\sim 1300 \mathrm{~h}$ after each medication exposure. Blood was drawn at $\sim 1400 \mathrm{~h}$ at baseline and after each scan to assess cortisol and phenytoin levels. The samples were sent to the Immunopharmacology Laboratory at the National Jewish Medical and Research Center in Denver, Colorado for cortisol analysis. Participants were paid for their participation in the study.

\section{Scan Acquisition}

MRI scans were performed on a 3 Tesla Philips Achieva system (Philips Medical Systems, Best, the Netherlands). A body coil was used for radiofrequency transmission and an eight-channel transmit/receive head coil was used for MRI signal reception. Foam padding stabilized the head and minimized motion. A T1-weight anatomic image was acquired using a MPRAGE (Magnetization-PreparedRapid-Acquisition-of-Gradient-Echo) sequence with the following imaging parameters: $\mathrm{TR}=8.14 \mathrm{~ms}, \mathrm{TE}=3.73 \mathrm{~ms}$, $\mathrm{TI}=1100 \mathrm{~ms}$, field-of-view $256 \times 256 \times 160 \mathrm{~mm}^{3}$, matrix $256 \times 256 \times 160$, voxel size $1 \times 1 \times 1 \mathrm{~mm}^{3}$, duration $3 \mathrm{~min}$ and $57 \mathrm{~s}$.

\section{Structural Imaging Analysis}

Structural MRI scans and cortisol levels were obtained following each medication condition. The total brain volume (TBV) and hippocampal volumes were manually traced by two trained and reliable raters on previously published protocols using BRAINS2 software (Magnotta et al, 2002). These raters were blind to participant identity, study group assignment, and clinical data. Inter-rater reliabilities (intraclass correlations; $n=10$ ) were 0.92 for the TBV, 0.91 for the right hippocampus, and 0.94 for the left hippocampus. Image data were resampled to align with anterior commissure posterior commissure, and then anatomic boundaries of the left hippocampus, right hippocampus, and TBV were traced manually using established methods, differentiating the amygdala and hippocampus (Shenton et al, 2001; Shenton et al, 2002). TBV was measured using a standard protocol, which includes the combined volume of the cerebral hemispheres, cerebellum, and the brainstem as well as the ventricular and extraventricular cerebrospinal fluid (Gilbert et al, 2001).

\section{Statistical Analysis}

Of the 17 participants, a total of 14 had complete data (no missed scans) and were used in the analysis. The order of treatments was randomized by a statistician using a random number sequence and balanced such that every treatment followed every other treatment the same number of times. Because of the crossover design, all effects were within subject. Therefore, a crossover $t$-test, which accounts for both treatment effect and sequence of drug administration, was used to compare mean volumes during each of the four treatment conditions, allowing for possible period effect-sequence of different treatment (Senn, 2006). Left and right hippocampal volumes were added together to create the total hippocampal volume values that were used in the analysis. Crossover $t$-tests examined each medication treatment differences in mean volumes of the manual traced total brain and hippocampus from placebo/ placebo condition-hydrocortisone/placebo vs placebo/ placebo, phenytoin/placebo vs placebo/placebo, and hydrocortisone/phenytoin $v s$ placebo/placebo. Pearson's correlation coefficient was used to analyze the correlations between changes in cortisol levels and brain volumes, as well as in declarative memory as assessed by the Rey Auditory Verbal Learning Test (RAVLT; Ryan et al, 1986). SAS 9.3 (SAS Institute, Cary, NC) was used for all the analyses. All tests were two-sided, and $P<0.05$ was used to assess the significance. 


\section{RESULTS}

Demographic and baseline characteristics of the participants are given in Table 1. The sample was relatively young and included more women than men. The study procedures were well tolerated.

TBV and total hippocampal data in the four treatment conditions are given in Table 2. The between-medication condition differences in TBV were small (mean 0.26\%) and nonsignificant between the hydrocortisone/placebo and placebo/placebo conditions. Hippocampal volume, however, showed a significant $1.69 \%$ mean decrease during the hydrocortisone/placebo condition as compared with the placebo/placebo condition $(t=-2.42, \mathrm{df}=12, P=0.03)$. The phenytoin/hydrocortisone $(t=-1.72, \mathrm{df}=11, P=0.11)$ and phenytoin/placebo $(t=0.78, \mathrm{df}=13, P=0.40)$ conditions were not significantly different in hippocampal volume from the placebo/placebo condition.

Given negative findings from a prior study with a similar design in young men (Tessner et al, 2007), and the mixed sample of men and women in our sample, we explored gender differences in the current study. In women $(n=9)$, a significant $2.01 \%$ reduction in mean total hippocampal volume was observed with hydrocortisone/placebo, as compared with placebo/placebo (treatment: $t=-2.39$; $P=0.048$, sequence: $t=-1.41 ; P=0.200)$, whereas in men $(n=5)$ a nonsignificant $0.81 \%$ reduction (treatment:

Table I Baseline Demographic Characteristics of Participants $(N=15)$

\begin{tabular}{lcc}
\hline & Mean or \% $(\mathbf{N}=\mathbf{I 5})$ & SD \\
\hline Variable & 25.3 & 8.1 \\
Age (years) & 16.5 & 2.8 \\
Education (years) & 60.0 & \\
Female (\%) & & \\
& & \\
Race & 73.3 & \\
Caucasian (\%) & 6.7 & \\
African American (\%) & 13.3 & \\
Hispanic (\%) & 6.7 & \\
Native American (\%) & 83.3 & \\
Right handed (\%) & 53.8 & \\
RAVLT total t-score & & \\
\hline
\end{tabular}

Abbreviation: RAVLT, Rey Auditory Verbal Learning Test. $t=-0.88 ; \quad P=0.444$, sequence: $t=-0.34 ; \quad P=0.7582$ ) was observed. Because a report suggested changes in hippocampal volume based on menstrual cycle phase (Protopopescu et al, 2008) we estimated the phase (luteal $v s$ follicular) at each assessment (based on the baseline reported last menstrual period and assuming a 28 day cycle). The numbers were very small but no significant difference in change in hippocampal volume was observed in women ( $n=8$ with menstrual phase data) who were in the same cycle phase $v s$ a change in phase between the placebo/placebo and hydrocortisone/placebo conditions $(t=0.66, P=0.533)$.

Cortisol levels increased following hydrocortisone administration (hydrocortisone/placebo-placebo/placebo, mean change $=19.79 \pm 20.86, \quad \mathrm{df}=14, \quad t=-3.61, \quad P=0.0025$; phenytoin/hydrocortisone - placebo/placebo, mean change $=$ $25.53 \pm 28, \mathrm{df}=14, t=-3.51, P=0.0034)$. The change in plasma cortisol levels between the placebo and hydrocortisone conditions correlated negatively with the change in hippocampal volume $(r=-0.58, P=0.031)$ (Figure 1$)$, but the correlation between the change in cortisol and the change in TBV was not significant $(r=0.15, P=0.617)$. Change in the RAVLT score (declarative memory) between the placebo and hydrocortisone conditions showed a moderate correlation $(r=0.42, P=0.130)$ that, perhaps owing to the small sample size, did not reach statistical significance.

\section{DISCUSSION}

We previously reported $8-9 \%$ smaller hippocampal volume in a clinical sample of medical patients receiving prescription prednisone therapy at a mean dose of $\sim 15 \mathrm{mg}$ /day, for a mean duration of about 8 years (Brown et al, 2004). The current report suggests that corticosteroid administration to healthy volunteers is associated with a significant reduction in hippocampal volume after only 3 days. This is a new and somewhat startling finding, suggesting that corticosteroids are associated with rapid changes in hippocampal structure in humans. The relationship between the change in hippocampal volume and the change in cortisol levels during hydrocortisone administration strongly suggests that the change in volume was owing to the effects of cortisol. The absence of a significant sequence effect (that is, whether hydrocortisone or placebo was administered first) suggests that the changes in hippocampal volume were reversible during the washout period. The findings have clinical significance. Prior research has demonstrated reversible

Table 2 Hippocampal and Total Brain Volume Differences Between Treatment Conditions

\begin{tabular}{llcllrr}
\hline Brain region & Difference & Mean $\boldsymbol{\Delta}\left(\mathbf{m m}^{\mathbf{3}}\right)$ & STD & DF & T-value & P-value \\
\hline Hippocampus & Phen/HC-Pbo/Pbo & -0.05 & 0.12 & 11 & -1.72 & 0.11 \\
Hippocampus & Phen/Pbo-Pbo/Pbo & -0.03 & 0.17 & 13 & -0.78 & 0.45 \\
Hippocampus & Pbo/HC-Pbo/Pbo & -0.12 & 0.17 & 12 & -2.42 & 0.03 \\
TBV & Phen/HC-Pbo/Pbo & -1.72 & 4.25 & 11 & -1.43 & 0.18 \\
TBV & Phen/Pbo-Pbo/Pbo & -0.03 & 0.17 & 13 & -0.78 & 0.45 \\
TBV & Pbo/HC-Pbo/Pbo & -3.48 & 4.35 & 12 & -0.43 & -0.40 \\
\hline
\end{tabular}

Abbreviation: TBV, total brain volume. 


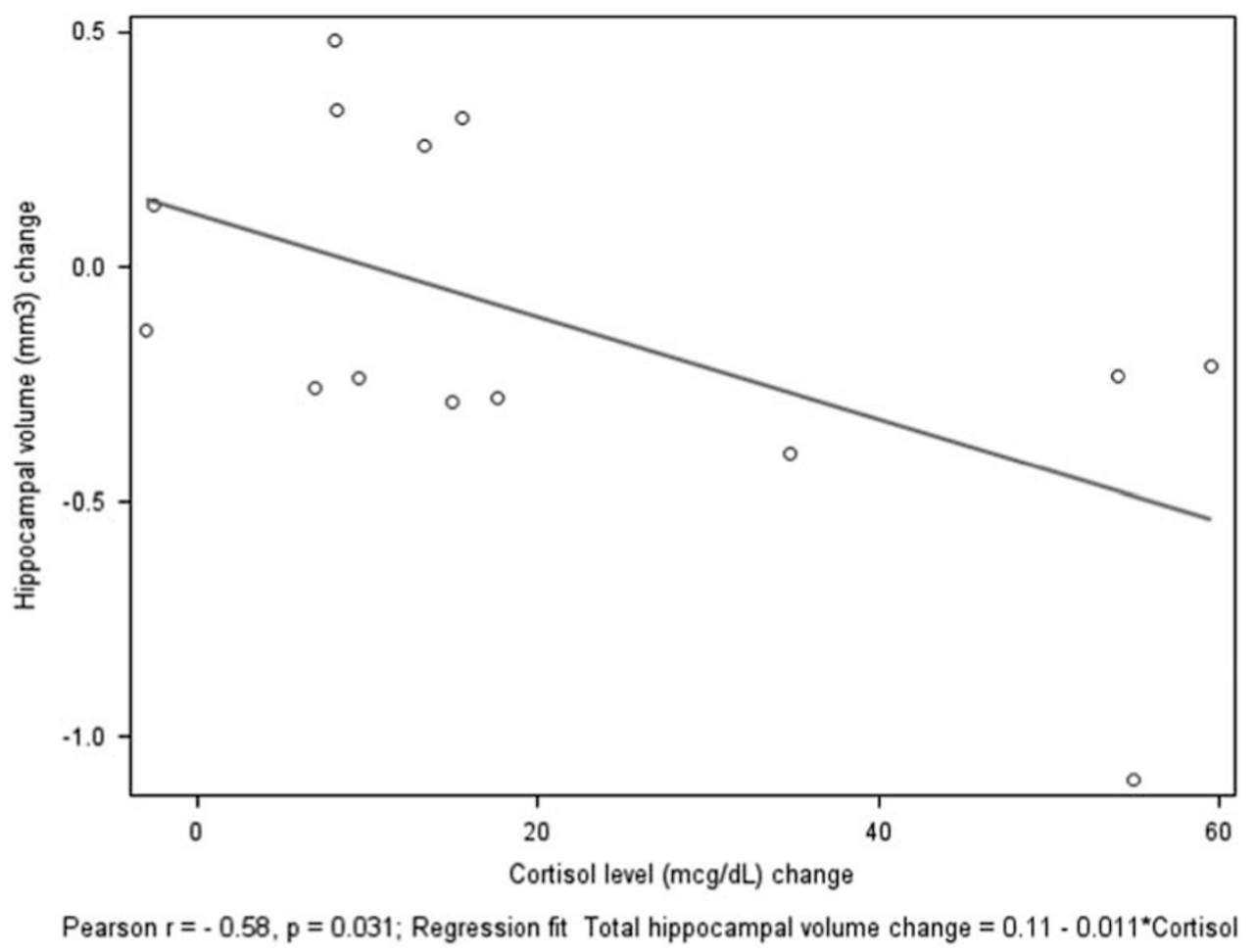

Figure I Change in hippocampal volume vs change in cortisol level in the hydrocortisone/placebo-placebo/placebo conditions.

changes in the declarative memory following very brief corticosteroid administration (Brown et al, 2006; Newcomer et al, 1999). The current findings suggest that hippocampal size may also decrease during several days of stress or during corticosteroid 'bursts' for allergies, dermatologic conditions, or asthma exacerbations.

Although mechanisms such as neuronal death (Reagan and McEwen, 1997; Uno et al, 1989) or a reduction in hippocampal neurogenesis (Saaltink and Vreugdenhil, 2014) might explain a reduction in hippocampal volume during extended exposure to exogenous corticosteroids, the observed rapid and reversible changes in hippocampal volume in the current report suggest that other mechanisms may be involved. Corticosteroids are sometimes administered for cerebral edema, and decreases in brain water with corticosteroids have been reported with magnetic resonance spectroscopy (Chumas et al, 1997). Reduction in cerebral edema with corticosteroids in patients with brain tumors may be mediated through a reduction in cerebral blood flow (Behrens et al, 1998). Thus, one possibility is that hippocampal neurons decreased in size owing to a reduction in the cellular water content. However, it is important to note that no change in TBV was observed. If changes in neuronal water content explain the observed findings, these changes must be more robust in the hippocampus than the brain as a whole. In animal models, the shrinkage of hippocampal dendrites and their spines has been reported during stress paradigms. A similar process may be occurring in humans during brief exposure to corticosteroids. The hippocampus is a brain region with a high concentration of GR receptors. Therefore, the specificity of the volume reduction to the hippocampus, rather than the total brain, is consistent with a process mediated through GR receptors. Thus, the mechanism may involve a genomic mechanism with membrane GR receptor binding, translocation to the nucleus and mRNA transcription. However, the rapid changes observed might also be suggestive of the non-genomic effects through membrane MR receptors (Reul and de Kloet, 1985).

Two prior studies examining the short-term exposure to corticosteroids did not demonstrate a change in hippocampal volume. In one of these studies, only a single dose of hydrocortisone was administered and the dose given was much lower than in the current study (Tessner et al, 2007). However, another study gave the same hydrocortisone dose as in the current report for 4 days (Scheel et al, 2010). Because these studies only included men, whereas our study included both men and women, we examined the gender differences in response. Although this subgroup analysis was limited by the small numbers in each group, the findings suggest that women may show a larger reduction in hippocampal volume with 3 days of hydrocortisone exposure than men. Research on gender differences in the cognitive response to brief stress or corticosteroid administration suggest a greater cognitive change in women (Almela et al, 2011; Breitberg et al, 2013); however, a stronger relationship between stress-induced cortisol elevation and the change in memory in men than in women has also been reported (Wolf et al, 2001). In addition, a large study in a clinical population suggested that men were more likely than women to develop confusion or delirium with prescription corticosteroids (Fardet et al, 2012). In animal models, the limited available data suggest that female rats may demonstrate a greater reduction in hippocampal dendrite density than male rats following a single stressor 
(Shors et al, 2001), but demonstrate less dentritic shortening than males in response to more chronic, 21-day, stress (Galea et al, 1997). Thus, it is important to consider possible gender differences in clinical and preclinical research involving stress or corticosteroid exposure.

The findings in the study have clinical as well as mechanistic implications. Corticosteroids are commonly prescribed medications that are associated with a variety of mood and cognitive changes (Bender et al, 1988; Fardet et al, 2012; Judd et al, 2014; Sternberg and Judd, 2009). The current findings suggest that changes in hippocampal structure may also occur during brief corticosteroid bursts used to treat asthma, allergies, and dermatological conditions.

It is striking that the effect of hydrocortisone on hippocampal volume is attenuated by the pretreatment with phenytoin. This finding is consistent with research in animal models demonstrating that phenytoin blocks hippocampal dendritic shortening during a chronic stress paradigm (Watanabe et al, 1992). We previously demonstrated that phenytoin pretreatment blocked the declarative memory effects, but potentiated the reduction in hippocampal activation (BOLD signal), of hydrocortisone (Brown et al, 2013). The current findings suggest that hippocampal volume may be a more targeted biomarker of the ability of phenytoin to block the effects of hydrocortisone on the hippocampus than was the BOLD signal. Phenytoin has a complex mechanism of action that includes reducing glutamate release. The reduction in glutamate release with phenytoin is thought to be the mechanism responsible for its ability to prevent stress-induced dendritic changes in animal models (McEwen et al, 1997). If this assumption is accurate then the changes in hippocampal volume observed with hydrocortisone in the present study may be owing to the effects of glutamate. Phenytoin was used in this paradigm to replicate preclinical findings in humans. These preliminary findings are not sufficient to recommend routine pretreatment with phenytoin in corticosteroidtreated patients. Although a brief pretreatment with phenytoin in the current study did not result in a decrease in cortisol levels, longer-term exposure might lower corticosteroid levels and possibly decrease their clinical effectiveness (Petereit and Meikle, 1977). The findings do, however, suggest that glutamate release inhibitors with a more favorable side effect and drug-drug interaction profile should be explored for preventing or reversing corticosteroid-induced changes in the hippocampus. For example, we reported that lamotrigine may improve declarative memory in patients taking long-term prescription corticosteroid therapy (Brown et al, 2008).

The study has limitations. The sample size of this pilot study is fairly small. Nonetheless, the ability to detect hippocampal changes with hydrocortisone was enhanced by the within-subject design. Carryover effects are a concern with a crossover design. However, the washout period was relatively long and we previously demonstrated that declarative memory changes with corticosteroids return to baseline within this timeframe (Brown et al, 2006). Because brain volumes were manually traced a possible source of error could be the accuracy and consistency of the tracings. To minimize this potential problem, extensive training and supervision of the persons performing the analysis was provided and good inter-rater reliability established.
In summary, a significant reduction in hippocampal volume that correlated with changes in cortisol level was observed following 3 days of hydrocortisone administration in healthy controls demonstrating that structural changes in the hippocampus can be observed very quickly following corticosteroid exposure. Although somewhat speculative at this point, the findings could have implications for better understanding the response of the hippocampus to acute stress as well as to patients taking brief bursts of prescription corticosteroids. Phenytoin pretreatment prevented these hippocampal volume changes following hydrocortisone administration. Additional research is needed to replicate the findings from this pilot study, as well as to determine whether even shorter exposures to hydrocortisone elicit a similar change in hippocampal volume, and to elucidate the mechanisms responsible for these changes.

\section{FUNDING AND DISCLOSURE}

Dr Brown has research grant support from NIMH, NIAAA, NIDA, NHLBI, NCCAM, the Stanley Medical Research Institute, Forest, Sunovion, and Alkermes. Dr Tamminga reports consulting income from Astellas, Eli Lilly, IntraCellular Therapies, Kaye Scholer LLC, and Lundbeck, as well as income from NIMH (council member), and APA (deputy editor). The remaining authors declare no conflict of interest.

\section{ACKNOWLEDGEMENTS}

This study was funded by NIH grant MH078182.

\section{REFERENCES}

Almela M, Hidalgo V, Villada C, Espin L, Gomez-Amor J, Salvador A (2011). The impact of cortisol reactivity to acute stress on memory: sex differences in middle-aged people. Stress 14: 117-127.

Behrens PF, Ostertag CB, Warnke PC (1998). Regional cerebral blood flow in peritumoral brain edema during dexamethasone treatment: a xenon-enhanced computed tomographic study. Neurosurgery 43: 235-240 discussion 240-241.

Bender BG, Lerner JA, Kollasch E (1988). Mood and memory changes in asthmatic children receiving corticosteroids. J Am Acad Child Adolesc Psychiatry 27: 720-725.

Breitberg A, Drevets WC, Wood SE, Mah L, Schulkin J, Sahakian BJ et al (2013). Hydrocortisone infusion exerts dose- and sexdependent effects on attention to emotional stimuli. Brain Cogn 81: 247-255.

Brown ES (2009). Effects of glucocorticoids on mood, memory, and the hippocampus. Treatment and preventive therapy. Ann NY Acad Sci 1179: 41-55.

Brown ES, Beard L, Frol AB, Rush AJ (2006). Effect of two prednisone exposures on mood and declarative memory. Neurobiol Learn Mem 86: 28-34.

Brown ES, J Woolston D, Frol A, Bobadilla L, Khan DA, Hanczyc M et al (2004). Hippocampal volume, spectroscopy, cognition, and mood in patients receiving corticosteroid therapy. Biol Psychiatry 55: 538-545.

Brown ES, Lu H, Denniston D, Uh J, Thomas BP, Carmody TJ et al (2013). A randomized, placebo-controlled proof-of-concept, crossover trial of phenytoin for hydrocortisone-induced declarative memory changes. J Affect Disord 150: 551-558. 
Brown ES, Wolfshohl J, Shad MU, Vazquez M, Osuji IJ (2008). Attenuation of the effects of corticosteroids on declarative memory with lamotrigine. Neuropsychopharmacology 33: 2376-2383.

Chumas P, Condon B, Oluoch-Olunya D, Griffiths S, Hadley D, Teasdale G (1997). Early changes in peritumorous oedema and contralateral white matter after dexamethasone: a study using proton magnetic resonance spectroscopy. J Neurol, Neurosur Psychiatry 62: 590-595.

Fardet L, Petersen I, Nazareth I (2012). Suicidal behavior and severe neuropsychiatric disorders following glucocorticoid therapy in primary care. Am J Psychiatry 169: 491-497.

First MB, Spitzer RL, Gibbon M, Williams JBW (1995). Structured Clinical Interview for DSM-IV Axis I Disorders. Biometrics Research Department, NY State Psychiatric Institute: New York, NY, USA.

Galea LA, McEwen BS, Tanapat P, Deak T, Spencer RL, Dhabhar FS (1997). Sex differences in dendritic atrophy of CA3 pyramidal neurons in response to chronic restraint stress. Neuroscience 81: 689-697.

Gilbert AR, Rosenberg DR, Harenski K, Spencer S, Sweeney JA, Keshavan MS (2001). Thalamic volumes in patients with firstepisode schizophrenia. Am J Psychiatry 158: 618-624.

Hamilton M (1960). A rating scale for depression. J Neurol Neurosur Psychiatry 23: 56-62.

Judd LL, Schettler PJ, Brown ES, Wolkowitz OM, Sternberg EM, Bender BG et al (2014). Adverse Consequences of Glucocorticoid Medication: Psychological, Cognitive, and Behavioral Effects. AmJ Psychiatry 171: 1045-1051.

Leverenz JB, Wilkinson CW, Wamble M, Corbin S, Grabber JE, Raskind MA et al (1999). Effect of chronic high-dose exogenous cortisol on hippocampal neuronal number in aged nonhuman primates. J Neurosci 19: 2356-2361.

Magarinos AM, Verdugo JM, McEwen BS (1997). Chronic stress alters synaptic terminal structure in hippocampus. Proc Natl Acad SciUSA 94: 14002-14008.

Magnotta VA, Harris G, Andreasen NC, O'Leary DS, Yuh WT, Heckel D (2002). Structural MR image processing using the BRAINS2 toolbox. Comput Med Imaging Graph 26: 251-264.

McEwen BS, Conrad CD, Kuroda Y, Frankfurt M, Magarinos AM, McKittrick C (1997). Prevention of stress-induced morphological and cognitive consequences. Eur Neuropsychopharmacol 7(Suppl 3): S323-S328.

Newcomer JW, Selke G, Melson AK, Hershey T, Craft S, Richards K et al (1999). Decreased memory performance in healthy humans induced by stress-level cortisol treatment. Arch Gen Psychiatry 56: $527-533$.

Petereit LB, Meikle AW (1977). Effectiveness of prednisolone during phenytoin therapy. Clin Pharmacol Ther 22: 912-916.

Protopopescu X, Butler T, Pan H, Root J, Altemus M, Polanecsky M et al (2008). Hippocampal structural changes across the menstrual cycle. Hippocampus 18: 985-988.

Reagan LP, McEwen BS (1997). Controversies surrounding glucocorticoid-mediated cell death in the hippocampus. J Chem Neuroanat 13: 149-167.
Reul JM, de Kloet ER (1985). Two receptor systems for corticosterone in rat brain: microdistribution and differential occupation. Endocrinology 117: 2505-2511.

Ryan JJ, Geisser ME, Randall DM, Georgemiller RJ (1986). Alternate form reliability and equivalency of the Rey Auditory Verbal Learning Test. J Clin Exp Neuropsychol 8: 611-616.

Saaltink DJ, Vreugdenhil E (2014). Stress, glucocorticoid receptors, and adult neurogenesis: a balance between excitation and inhibition? Cell Mol Life Sci 71: 2499-2515.

Sapolsky RM, Uno H, Rebert CS, Finch CE (1990). Hippocampal damage associated with prolonged glucocorticoid exposure in primates. J Neurosci 10: 2897-2902.

Scheel M, Strohle A, Bruhn H (2010). Effects of short-term stress-like cortisol on cerebral metabolism: a proton magnetic resonance spectroscopy study at 3.0 T. J Psychiatr Res 44: $521-526$.

Senn S (2006). Cross-over trials in statistics in medicine: the first '25' years. Stat Med 25: 3430-3442.

Shenton ME, Dickey CC, Frumin M, McCarley RW (2001). A review of MRI findings in schizophrenia. Schizophr Res 49: 1-52.

Shenton ME, Gerig G, McCarley RW, Szekely G, Kikinis R (2002). Amygdala-hippocampal shape differences in schizophrenia: the application of 3D shape models to volumetric MR data. Psychiatry Res 115: 15-35.

Shors TJ, Chua C, Falduto J (2001). Sex differences and opposite effects of stress on dendritic spine density in the male versus female hippocampus. J Neurosci 21: 6292-6297.

Starkman MN, Gebarski SS, Berent S, Schteingart DE (1992). Hippocampal formation volume, memory dysfunction, and cortisol levels in patients with Cushing's syndrome. Biol Psychiatry 32: 756-765.

Sternberg EM, Judd LL (2009). Conference summary and conclusions. A comprehensive approach to predicting and managing mood effects of glucocorticoids. Ann NY Acad Sci 1179: 229-233.

Tessner KD, Walker EF, Dhruv SH, Hochman K, Hamann S (2007). The relation of cortisol levels with hippocampus volumes under baseline and challenge conditions. Brain Res 1179: 70-78.

Uno H, Eisele S, Sakai A, Shelton S, Baker E, DeJesus O et al (1994). Neurotoxicity of glucocorticoids in the primate brain. Horm Behav 28: 336-348.

Uno H, Tarara R, Else JG, Suleman MA, Sapolsky RM (1989). Hippocampal damage associated with prolonged and fatal stress in primates. $J$ Neurosci 9: 1705-1711.

Vyas A, Mitra R, Shankaranarayana Rao BS, Chattarji S (2002). Chronic stress induces contrasting patterns of dendritic remodeling in hippocampal and amygdaloid neurons. J Neurosci 22: 6810-6818.

Watanabe Y, Gould E, Cameron HA, Daniels DC, McEwen BS (1992). Phenytoin prevents stress- and corticosterone-induced atrophy of CA3 pyramidal neurons. Hippocampus 2: 431-435.

Wolf OT, Schommer NC, Hellhammer DH, McEwen BS, Kirschbaum C (2001). The relationship between stress induced cortisol levels and memory differs between men and women. Psychoneuroendocrinology 26: 711-720. 